Revista de Indias, 1999, vol. LIX, núm. 217

\title{
UN LARGO CAMINO DE LÁGRIMAS: LA POLÍTICA INDIA DE LOS ESTADOS UNIDOS DE AMÉRICA
}

POR

\author{
FERNANDO MONGE
}

Centro de Estudios Históricos, CSIC

Concebido como un panorama general introductorio sobre la política india en los Estados Unidos, este artículo es también un ensayo que pretende mostrar cómo el contexto histórico, así como el origen, evolución y construcción del estado, contribuyeron al diseño de esa política.

Los objetivos que pretende cubrir este artículo son varios. En primer lugar trata de presentar un panorama de los antecedentes, origen y desarrollo de la política india en los Estados Unidos hasta la actualidad. Lo he diseñado como un ensayo que permita al lector trazar comparaciones con el resto de los artículos de este volumen monográfico. Por ello este marco introductorio se ciñe a presentar de un modo muy condensado los elementos que he considerado claves para adentrarse en un problema de una gran complejidad ${ }^{1}$.

Pretendo, en segundo lugar, mostrar que la política india no es el producto de los «expertos» en ese tema sino que surge y se desarrolla dentro de lo que es la propia génesis, formación y evolución de los Estados Unidos. De hecho ésta se inscribe, al igual que la propia política india, dentro de un marco mayor que es el de la propia evolución socio-cultural de la nación sobre la que estamos trabajando. Así, un tratamiento exhaustivo de la política india debe encuadrarse en el momento histórico en el que se está produciendo y, a ser posible, desde una pers-

1 Para aquel que quiera ampliar la información que le ofrezco he incluido algunas referencias comentadas en las notas a pie de página. El manual básico y clásico para adentrarse en este mundo es el monumental y todavía inconcluso Handbook of North American Indians, cuyo editor general es William C. STURTEVANT (se trata de una obra de veinte volúmenes). Para este tema en concreto el volumen más adecuado es el cuarto: History of Indian-White Relations, editado por Wilcomb E. WASHBURN, Washington, DC, Smithsonian Institution, 1988. Otro texto de gran utilidad es el de Carl Waldman, Atlas of the North American Indian [con mapas e ilustraciones de Molly Braun], Nueva York, Facts on File Publications, 1985. 
pectiva sensible, no sólo a los valores de quienes la ejecutaban y la sociedad dominante que la apoya, sino de la perspectiva de los sometidos a esa política.

Tanto el primer objetivo, el panorama general, como el ensayo de contextualización de la política india de los Estados Unidos hacen, en mi opinión, necesario incluir una tercera dimensión u objetivo a mi artículo: la construcción de una imagen del indio que unifique en uno sólo el grupo «indio» a los múltiples grupos o naciones nativas con las que se encontraron los colonizadores en su avance desde las costa este de Norteamérica hacia el oeste ${ }^{2}$. De hecho, es este proceso de colonización y consolidación de un estado independiente de los poderes europeos el que acabaría por gestar una política india diseñada para unos nativos que sólo con el paso del tiempo y la aplicación de esa política comenzarían a reconocerse como «indios» a finales del siglo XIX. Dicha política india no pudo surgir, tal como pretendo mostrar, antes de que existiera un estado federal estructurado ni tampoco antes que los indios se reconocieran como tales en vez de sioux, creek, seneca, etc. Existieron, sin lugar a dudas, múltiples ensayos de ámbito más reducido, así como intentos por parte de la metrópoli británica de introducir una normativa general. Ahora bien, éstos no pueden ser entendidos sin tener en cuenta cúal fue su alcance real ${ }^{3}$.

2 Aunque los franceses, españoles, holandeses y suecos que estuvieron son también protagonistas de la conquista de lo que serian los Estados Unidos, he considerado conveniente centrarme en el grupo que consiguió imponer su dominio no sólo sobre los distintos grupos nativos sino europeos.

3 Hace unos años los manuales sobre «indios de América del norte» solían centrarse en la descripción de las características de los numerosos grupos y/o naciones nativas, agrupadas por áreas culturales y congeladas en un pasado histórico habitualmente inmediatamente previo al contacto directo con los blancos. Solían incluir algín capítulo al final sobre las relaciones entre nativos y blancos, como el clásico Indians of North America de Harold E. DRIVER, University of Chicago Press, Chicago, 1969 (segunda edición revisada). Entre este tipo de manuales destacó en su día la obrita de D'Arcy MCNICKLE, Las tribus indias de los Estados Unidos (subtitulada en inglés: Ethnic and Cultural Survival), Editorial Universitaria de Buenos Aires, Buenos Aires, 1965 (ed. orig. 1962). Hoy, al elaborar un manual sobre los nativos de Estados Unidos o Canadá, no se olvida su historia y situación actual. Gozamos de varios excelentes ejemplos publicados en castellano: J. Anthony PAREDES, Indios de los Estados Unidos Anglosajones, Mapfre, Madrid, 1992; Thomas WEAVER, Los indios del gran suroeste de los Estados Unidos, Mapfre, Madrid, 1992; Bruce Alden Cox, Los indios del Canadá, Mapfre, Madrid, 1992; Ramón Hernando DE LaRramendi, Esquimales, Mapfre, Madrid, 1992. Una introducción en castellano al proceso de colonización que, aunque los cánones clásicos de análisis pretende ser más sensible al «factor indio» en su análisis es: Margarita Del Olmo y Fernando MONGE, Historia etnológica de los indios norteamericanos, Madrid, Akal, 1992. Un clásico estudio del efecto sobre los indios de una zona de los hoy Estados Unidos es el de Edward H. SPICER, Cicles of Conquest. The Impact of Spain, Mexico, and the United States on the Indians of the Southwest, 1533-1960, University of Arizona Press, Tucson, 1962. Sobre el renacimiento e historia de uno de las naciones iroquesas, véase: Anthony F.C. WALLACE, The Death and Rebirth of the Seneca, Vintage Books, Nueva York, 1972 (1 ed. 1969). Desde la perspectiva francesa y para el caso del noreste americano (fundamentalmente Canadá) ver: Denys DELAGE, Le pays renversé. Amérindiens et européens en Amérique du nord-est, 1600-1664, Boréal Express, Montreal, 1985.

R. I., $1999, \mathrm{n}^{\circ} 217$ 


\section{LA ETAPA BRITÁNICA}

Las sociedades de origen británico habían prosperado en Norteamérica gracias a una actividad privada refrendada por las concesiones de la Corona. Su relación con la metrópoli estaba lejos de ser sencilla o claramente pautada. Existían distintos tipos de colonias con distintas cartas fundacionales y estatutos jurídicos que trataban de asentarse y prosperar a lo largo de la costa este de Norteamérica. No sólo los marcos legales eran distintos, el propio entorno físico en el que intentaban prosperar las colonias, los deseos de crear sociedades libres de la corrupción de la que huían y las propias alternativas de desarrollo económico, hacían que las Trece Colonias se asemejaran en poco unas a otras. Sin embargo, todas estaban constituidas por pequeñas poblaciones que dependían de bienes manufacturados en las islas británicas y que, en lo político, apenas sufrieron intervención alguna por parte de la metrópoli hasta la segunda mitad del siglo XVIII, cuando se inició un ambicioso plan de reformas tributarias e imposición de leyes de navegación que conducirán eventualmente a la Guerra Revolucionaria e Independencia de los Estados Unidos de América.

Desde el punto de vista de sus relaciones con los nativos, las colonias consideraban a los grupos indios como a otra «raza» o nación. No contaban en sus proyectos coloniales más que como una población marginal con la que podía entablarse una relación comercial y de alianza que sirviera de colchón de seguridad frente a los ataques de otras «naciones» más peligrosas de cara a los colonos, como eran las colonias establecidas por coronas rivales, y además, como legitimadores de unos títulos de propiedad de la tierra apenas legalizados por las cartas de concesión real. El mecanismo lógico y más adecuado para mantener este marco de relaciones era, sin lugar a dudas, el tratado (internacional) entre naciones. Para la metrópoli el uso de la figura del tratado era también conveniente, y sus propios intereses hegemónicos y de control sobre las coronas rivales coincidían con los de los colonos, de modo que una política adaptada a las peculiaridades de cada caso era, por lo general, válida también para el mantenimiento del primer Imperio Británico.

Sin embargo, cuando con el Tratado de París de 1763 finalizó la Guerra de los Siete Años, que en Norteamérica se conoció como Guerra Franco India (1756-63), la corona británica se vio con la posibilidad de imponer una nueva política más restrictiva para los gobiernos coloniales. El esfuerzo que la metrópoli había hecho para derrotar a los franceses, tanto a un lado como al otro del Atlántico, tenía que verse compensado con el pago por las Trece Colonias de parte de los gastos de la guerra. Los colonos, por su parte no sólo no estaban de acuerdo, sino que pensaban que eran ellos quienes habían puesto en peligro o sacrificado su vida y posesiones para luchar contra los franceses del Canadá. Dicho conflicto, que generó como es bien sabido la espiral de desencuentros que

R. I., $1999, \mathrm{n}^{\circ} 217$ 
llevarían a la independencia de los Estados Unidos, también supuso una transformación importante de la actitud metropolitana hacia los «nativos».

Los graves problemas que distintas alianzas de grupos nativos generaban cíclicamente se debían, sin duda, al fortísimo incremento de población de colonos británicos en el área. La demanda de tierras era incesante y, con ella, los problemas. La corona británica, única poseedora ahora de ese área de Norteamérica, decidió imponer la primera normativa general relacionada con los indígenas: la Proclamación Real o Royal Proclamation de 1763. Hasta entonces los únicos elementos legales unificados aplicables eran las Actas de Navegación (16511673). Se habían creado además, en las mismas fechas, un cuerpo de aduanas colonial (1671) y una Comisión de Comercio y Plantaciones (Board of Trade and Plantations) destinadas a regular y limitar la «excesiva» autonomía de unas colonias a las que se les había dejado demasiado descontroladas.

La Proclamación Real de 1763, impuesta casi cien años después del inicio de la política de mayor control metropolitano ${ }^{4}$ sobre las Trece Colonias, pretendía solventar los conflictos con las naciones nativas, reconociendo los derechos de éstas a su territorio y estableciendo una línea de división entre las áreas en que los colonos podían asentarse y la zona de propiedad exclusiva de los nativos. Era territorio nativo todo aquel que se encontrara al oeste de la línea de cumbres de los Apalaches. Se creaban, asimismo, dos Departamentos de asuntos indios (con su respectivo superintendente, uno del norte y otro del sur) que regulaban el contacto comercial entre nativos y blancos en el área. Y supuso también, según los colonos, una de las quiebras de la autonomía política a la que tenían derecho y finalizó con la etapa de gobierno metropolitano caracterizada por la «negligencia benigna» al tratar de imponer ésta las teorías mercantilistas en su propio imperio 5 .

La radical decisión de segregación entre un área abierta a la colonización y otra cerrada no podía ser bien recibida por los colonos deseosos y necesitados de tierras. De hecho, no sólo introducía un nuevo tipo de interlocutor y marco legal de relación entre los nativos y los colonos, sino que, además, vetaba el desarrollo (al menos así lo entendían los colonos) de unas colonias y colonos que habían luchado por su rey y su tierra. De este modo, una medida diseñada con el objetivo de cerrar un periodo de crisis y crear un marco estable y segregado de relaciones entre los nativos y los colonos, no sirvió nada más que para aumentar la creciente lista de agravios de los británico-americanos con respecto a su metrópoli e introducir nuevas complicaciones, a las ya de por sí deterioradas relaciones entre

\footnotetext{
4 Dicha política de control creciente sobre las colonias inglesas de América y el Caribe se inició con el ascenso al trono de Jaime II en 1685.

$5 \mathrm{Si}$ bien los sucesos a los que me refiero son bien conocidos, menos son, sin embargo, los principios políticos y las interpretaciones divergentes del mismo que llevaron a los conflictos. Un excelente análisis de los mismos es A. PAGDEN Señores de todo el mundo. Ideologías del imperio en España, Inglaterra y Francia (en los siglos XVI, XVII y XVIII), Barcelona, Ediciones Península, 1997, en especial el capítulo 5: «Metrópoli y colonia», pp. 165-200.
} 
unos nativos capaces de elegir sus aliados y unos colonos que ya no se sentían en modo alguno dependientes de un clima de entendimiento con los propios nativos.

Si nos aproximamos al desarrollo de la política indígena desde una perspectiva espacial con el objeto de hacer más inteligible la evolución de la relación entre nativos y blancos a lo largo del periodo de colonización, aparece, dentro de la variabilidad de las experiencias que cada uno de los grupos nativos vivió durante más de dos siglos, un patrón de relación entre ambos mundos. El encuentro, tal como desarrolla Donald W. Meinig en su perspectiva geográfica de la formación de los Estados Unidos 6 , se produjo como contacto más o menos inesperado y, sobre todo, como conflicto ya que, si bien en las primeras fases de relación primó el interés por llevar adelante una relación pacífica, el resultado inevitable terminaría siendo un tremendo enfrentamiento entre las culturas, así como un choque medioambiental y una transformación posterior. La magnitud de los cambios que se estaban produciendo no podían, como señala el mismo autor, ser controladas por poder alguno, de hecho, escapaban a la propia capacidad de predicción de las partes involucradas. En este marco, más que la propia acción de la metrópoli lo que importaba era el nivel de relación local y es en él donde tenemos que buscar las fórmulas «políticas» que una parte, y la otra también, trataban de imponer o negociar.

La frontera que se iba abriendo desde la costa este hacia el interior, si puede considerararse así, era móvil y, en realidad, determinaba la existencia de un «tiempo» o contexto histórico determinado. Así, el patrón que diversos especialistas han marcado en la colonización desde el este de los Estados Unidos por parte de los entonces súbditos británicos, se iba poniendo en marcha en distintas regiones sucesivamente. Meinig sostiene que la colonización hasta 1800 puede estructurarse espacialmente en tres regiones que, hacia 1750, existían simultáneamente en las Trece Colonias. Dichas zonas avanzaban más hacia el interior, es decir, longitudinalmente, que de norte a sur ${ }^{7}$.

En la primera de las zonas, la sujeta a una colonización más intensa, la población nativa apenas existía o, la que todavía se mantenía estaba confinada en reservas. La pérdida de población masiva, provocada en gran medida por las epidemias, guerras y radical transformación del medio del que dependían muchas de las culturas originarias, habían llevado a que el equilibrio de poderes se hubiese inclinado definitivamente a favor de los colonos. La supervivencia para los nativos en este entorno social y medio ambiental los llevaba inexorablemente, sin que por ello perdieran su propia identidad, a hacerlos depender de las tecnologías importadas y a relacionarse con la cada vez más consolidada población «mayoritaria».

6 Donald W. MeInIG, The Shaping of America. A Geographical Perspective on 500 Years of History. Volume I: Atlantic America, 1492-1800., New Haven, Conn., Yale University Press, 1986, págs. 205-13.

7 Sigo aquí la división en zonas y parte de las ideas que Meinig desarrolla en el libro mencionado en la nota anterior, pp. 205-13. 
Más allá de este área se extendía la segunda zona. En ella dominaban las relaciones de interacción e interdependencia entre blancos y nativos. Un nuevo territorio iba estructurándose en torno a nuevos usos y patrones de relaciones intergrupales, nativos y blancos incluidos. En esta zona se iba extendiendo un patrón de asentamiento humano difuso, con comercio en los márgenes de los distintos territorios de predominancia y una creciente red de asentamientos urbanos, como Montreal, Albany, Charles Town o Mobile, que se iban convirtiendo en mercados especializados y complejos de contacto y articulación intercultural. Un medio como el que describo fue más propicio a los cambios mutuos, cierto nivel de mezcla y transformación de las identidades previas al contacto.

Por último, existía una tercera zona, más allá del encuentro habitual entre nativos y europeos donde se mostraban los procesos que debieron llevarse a cabo en la costa tras los primeros desembarcos de colonos. Un complejo patrón de atracción y repulsión funcionaba aquí. Las transformaciones en este entorno eran rápidas y dramáticas, nuevas alianzas, guerras y conflictos constantes dominados por los intereses locales de las fuerzas en contacto.

Las regiones definidas por Meinig, que organizan de un modo muy claro los distintos rasgos dominantes dentro de la tremenda variabilidad existente en el proceso de contacto entre los nativos y los blancos, tienen, sin duda, un claro componente secuencial e histórico que muestra, a diferencia de algunas de las obras puramente históricas dedicadas al mismo tema, la «verdad largamente omitida de que los indios estuvieron involucrados, de un modo crítico, en la creación de cada una de las colonias europeas en el continente» ${ }^{8}$. Por otra parte, organiza en torno a una serie de patrones ideales la tremenda variabilidad que el contacto y ulterior colonización produjo9. De aquí que, a la hora de trazar la constitución, el marco de relaciones entre los nativos y los europeos primero y una política india unificada después hace absolutamente necesario, incluso, introducir estos elementos en un panorama general sobre la política india en los Estados Unidos.

A menudo se ha insistido en los intereses económicos, obvios por otra parte, y el racismo que impulsó una política tan claramente dañina para los nativos. Sin embargo, al abordar el análisis de la política india en ese país se infravalora no

8 D.W Meinig [6], p. 213.

9 Desde una perspectiva más anclada en lo cronológico, Margarita del Olmo y Fernando Monge [3], desarrollaron un patrón en cinco fases de la colonización: 1. descubrimiento, 2. llegada de los colonizadores, 3 . entente comercial entre las colonias y los indios, 4. consolidación de la colonización europea, y 5 . la carrera hacia el oeste. Se corresponderían aproximadamente con las tres zonas del siguiente modo: primera zona - etapa 4: segunda zona - etapa 3 fundamentalmente; y tercera zona - etapa 2 . El hecho de que nuestra visión sea de mayor duración que la que Meinig desarrolla explicaría la exitencia de una primera etapa, el descubrimiento, en el que se desarrollan unos fuertes estereotipos del otro: y una quinta que obedece a un proceso posterior, semejante al que describe Meinig [6] para 1492 a 1800, pero en el que la predominancia europea es ya muy fuerte. 
sólo el papel del propio indígena en el proceso, sino que también se introducen numerosas deformaciones de índole cultural e histórica. En mi opinión, dichos sesgos se deben a la propia perspectiva y especialización a la que se han sometido los expertos en la política indígena y las relaciones entre los blancos y los nativos. Su orientación no es, como puede parecer por estas palabras, criticable en sí misma ${ }^{10}$ ya que los valores de lo realizado son innegables. Mi crítica se orienta, precisamente, a una falta por omisión, ya que la propia vitalidad de su perspectiva ha oscurecido alternativas de análisis que, como decía en la introducción, encuadren de un modo más sistemático la política india en la dinámica política general del país ${ }^{11}$, que tengan en cuenta, asimismo, el propio papel activo de los nativos y, por último, consideren la imagen e historia cultural con la que se construyó el «indio» desde el punto de vista de la sociedad dominante.

Por otra parte, desde la habitual perspectiva hispana se pierde de vista el «hecho diferencial» o excepcionalidad norteamericana, ya que las colonias británicas percibieron y construyeron su propia identidad en un abierto contraste político con las que, más al sur o al norte, constituyeron los españoles o franceses. Las colonias británicas habían surgido como un proyecto privado cuyo objetivo no era otro que el de crear una sociedad más perfecta y mejor que la que abandonaban. Formaban, como mencioné más arriba, parte del imperio, pero con una amplia autonomía política. Las relaciones con los nativos discurrieron, por tanto, de un modo doble.

En el día a día y en los problemas de constitución del espacio y sociedad colonial, el recurso al que se recurría se articulaba en torno un marco legal de origen europeo: el tratado, mientras que, para los grandes diseños imperiales era el rey el que, en primer lugar, emitía normativas y, en segundo, creaba instituciones que se ocupaban de los asuntos indígenas.

\section{LA CONSTRUCCIÓN DE LOS ESTADOS UNIDOS Y LA POLÍTICA INDIA.}

Desde el punto de vista de las relaciones con los indios, la demarcación de una línea de separación entre los colonos y los nativos en 1763 supuso un acto de buena voluntad de la corona británica que, cuando llegó la Guerra de la Indepen-

10 Un buen ejemplo de lo dicho es el excelente artículo de Lawrence C. KELLY, «United States Indian Policies, 1900-1980», en W.E. Washburn, ed., History of Indian-White Realations,, Volumen 4 del Handbook of North American Indians, Washington, D.C., Smithsonian Institution, 1988, pp. 66-80.

11 Dado que mucha de esta literatura se dirige a un lector que conoce bien la historia de los Estados Unidos, es posible que no se haya considerado necesario contextualizar en un proceso histórico más general sus textos.

R. I., $1999, \mathrm{n}^{\circ} 217$ 
dencia, alineó a la mayoría de los nativos del lado leal a la metrópolii12. El Congreso Continental de los colonos sublevados decidió instituir, por su parte, un Comité de Asuntos Indios a imagen y semejanza del creado por orden real, pero dividido en tres departamentos. Continuaba así la doctrina precedente de mantener el control de los asuntos indios en manos de la más alta instancia legislativa: el Congreso federal, en vez de en los estados surgidos de cada una de las colonias.

Los líderes revolucionarios mantuvieron, también, la doctrina de la soberanía de los grupos o naciones indias y, por lo tanto, continuó aplicándose la política de establecimiento de tratados que legalizaba la ocupación de territorios, así como el establecimiento de unos acuerdos de relación y contraprestaciones -como la definición de áreas de ocupación reservada o reservas, y la concesión por parte del Congreso de una serie de contraprestaciones, a cambio de la cesión de la soberanía de las tierras en las que tradicionalmente se habían asentado los grupos nativos con los que habían llegado a un acuerdo.

Los recién nacidos Estados Unidos, ante las dificultades que creaba la expansión de los colonos, ubicaron los asuntos indios bajo el mandato de la Secretaría de Guerra y, durante los próximos años, se instauraría una serie de leyes de comercio y relación que iban a desarrollar un sistema de factorías en territorio indio. En 1824, una vez desaparecida la Oficina de Comercio Indio, se creó la Oficina de Asuntos Indios (Bureau of Indian Affairs) en la Secretaría de la Guerra; y, en 1932, el Congreso ratificó esta estructuración institucional y concedió al presidente la facultad de nombrar un Comisario de Asuntos Indios. A esta unificación institucional le seguiría, dos años después, el Acta o Ley de Comercio y Relaciones [Trade and Intercourse Act]. Si atendemos al aspecto formal de estos hitos, para la cuarta década del siglos XIX ya se puede considerar que existía una «política india» estatal (en este caso federal). Ahora bien, si nos ceñimos, como anunciaba más arriba, a considerar dicha política como el producto de un contexto histórico y geográfico dado, así como la propia acción de los nativos, echaremos todavía en falta un elemento clave para constituir una «política india»: los indios.

12 Fundamento mi descripción de los acontecimientos así como algunas de las perspectivas de los párrafos que siguen en: C. WALdMan [1], W.E. Washburn [1] Y M. Del OLMO Y F. Monge [3]. Sigo aquí también, aunque con más precauciones, las ideas de Francis P. PRUCHA, The Indians in American Society. From the Revolutionary War to the Present, University of California Press, Berkeley, Calif., 1985. Éste es una compilación de cuatro conferencias que se sustentan en su reconocida carrera como experto en la historia india, así como su libro: The Great Father: The United States Government and the American Indians, University of Nebraska Press, Lincoln, 1984.

R. I., $1999, \mathrm{n}^{\circ} 217$ 
$\mathrm{El}$ «problema indio».

Los nativos que poblaban el vasto continente norteamericano que, con el tiempo, ocuparían los Estados Unidos, el Canadá13 y México, constituían un universo de naciones, culturas, lenguas y formas de vida en sí mismos. Más de quinientas naciones, como se definía en una popular serie divulgativa de televisión de los Estados Unidos, poblaban este inmenso territorio. Algunas de ellas estaban ya relacionadas por alianzas o confederaciones anteriores a la colonización, como la de las Cinco Naciones (luego seis) Iroquesas, otras lo hicieron por la propia presión de la penetración europea ${ }^{14}$ sin embargo, ninguna de ellas se consideraba perteneciente a esa categoría de «indio» con la que los blancos denominaban a todos los pueblos nativos de los dos continentes. Quizá por ello, como decía el líder sioux Vine Deloria, Jr:

«Los indios son como el tiempo. Se sabe todo sobre el tiempo pero nadie puede cambiarlo. Cuando se espera una tormenta, sale el sol. Cuando se anuncia que hará buen tiempo para salir a merendar al campo comienza a llover. De modo parecido, si contáis con la imprevisibilidad de los indios, nunca tendréis que arrepentiros.

Una de las cosas más hermosas de ser indio es que la gente siempre muestra interés por tu 'situación'. Los otros grupos tienen dificultades, apuros, aprietos, problemas o penas. Es tradicional que nosotros, los indios, tengamos una 'situación'.

Nuestra situación principal es ser transparentes» ${ }^{15}$.

13 Como referencia y orientación véase para Canadá: James S. FRIDERES, Native People in Canada. Contemporary Conflicts, Prentice-Hall Canada, Scarborough, Ontario, 1983 (2da ed.); J. Rick Ponting, ed., Arduous Journey. Canadian Indians and Decolonization, McClellan \& Stewart, Toronto, 1988; Sally M. WEAVER, Making Canadian Indian Policy. The Hidden Agenda, 1968-1970, University of Toronto Press, Toronto, 1981; Joan RYAN, Wall of Words. The Betrayal of the Urban Indian, PMA Books, Toronto, 1978; y para la costa oeste del Canadá: Robin FisHER, Contact and Conflict. Indian-European Relations in British Columbia, 1774-1890, University of British Columbia Press, Vancouver, BC, 1977; F.E. LAVIOLETTE, The Struggle for Survival. Indian Cultures \& the Protestant Ethic in British Columbia, University of Toronto Press, Toronto, 1973 (1 ed. 1961).

14 Muchas de las confederaciones que surgieron posteriormente, hasta la aparición de organizaciones pan-indias, fueron el producto coyuntural de alianzas militares contra los británicos, franceses, holandeses o, más al sur, españoles.

15 Vine DeloRIA, Jr., El general Custer murió por vuestros pecados. Un manifiesto indio, Barcelona, Barral, 1975 (ed. orig. 1969), p. 7. Véase, también, otros autores comprometidos con la lucha indígena durante esos años, como Alvin M. JOSEPHY, Jr., Red Power. The American Indians' Fight for Freedom, McGraw-Hill, New York, 1971 (una interesante recopilación de textos de los sesenta representativos de la lucha por los derechos indios desde su propia perspectiva). Del mismo autor: Now that the Buffalo's Gone. A Study of Today's American Indians, Alfred A. Knopf, New York, 1982 (este texto, aunque orientado desde la perspectiva de la lucha india de las décadas anteriores actualiza muchos de los problemas y cuestiones de la literatura que ya he mencionado).

R. I., 1999, n. $^{\circ} 217$ 
La «transparencia» a la que alude Deloria no es, quizás, más que la insistente superposición sobre su propia identidad de una imagen o concepción prefabricada por los europeos y colonos. Por eso, no sólo son «imprevisibles» y viven teniendo una «situación» que comprendemos y para la que se diseña una política de ayuda concreta. Sus propias perspectivas, una vez que se impusieron los colonizadores, de poco han contado hasta hace muy pocos años.

Por si ello fuera poco, la tremenda diversidad de formas de vida y estructuración social y política, por paradójico que parezca, no ayudaba mucho a la hora de reconstruir una imagen del nativo que se definía más por carencias que por rasgos positivos. La imagen del indio o el paradigma sobre éste, construido a modo de prejuicio en los primeros contactos de los nativos con los españoles, e iba a ser válido con modificaciones que en nada mejoraban el modo en que se les consideraba. Los colonos británicos no encontraron grandes ciudades o «civilizaciones» como los españoles. La definición por «carencias» hacía fácil que los indios fueran considerados como unos «otros» deficientes ${ }^{16}$. Si lo que destacaba eran sus faltas religiosas se les denominaba, además de indios, paganos, infieles o bárbaros; si eran rasgos generales como el entorno en el que vivían, era hombres de la selva o salvajes ...(su apariencia física, pese a ser muy variada en el color de la piel, les valió un término que les alejaba de los blancos, como era lógico esperar de un «otro», y les convertía en «pieles rojas», ahora bien, sin llegar a ser negros).

La concepción que los recién llegados tenían de los denominados «indios» no iba a transformarse gracias al relativamente reducido contacto directo de colonos con nativos. De hecho, como ya he apuntado, la relación se reducía fundamentalmente a la interacción comercial o la consecución de acuerdos. Si atendemos a las regiones de «encuentro» que definió Meinig ${ }^{17}$, o las fases de relación histórica que establecimos Margarita del Olmo y yo ${ }^{18}$, existe una evolución oscilante entre el «buen» y el «mal» salvaje que dependía de la existencia o no de conflictos entre los colonos y los nativos. De hecho, desde los primeros e inciertos momentos de colonización inicial en el que fueron los propios indígenas los que ayudaron a la supervivencia de los colonizadores (de donde surge la fiesta más importante de los EEUU: el Día de Acción de Gracias), se fue avanzando hacia una actitud cada vez más condescendiente y marginadora (siempre que no se estuviese viviendo alguna guerra contra los nativos).

Sobre este entramado conceptual, a menudo tan importante como el día a día entre ambos mundos y los intereses materiales de los colonizadores, se fue defi-

16 Una excelente síntesis e introducción a la concepción blanca de los indios es: Robert F. BERKHOFER, JR., «White Conceptions of Indians», en W.E. WASHBURN, ed., History of IndianWhite Relations, Volumen 4 del Handbook of North American Indians, Washington, D.C., Smithsonian Institution, 1988, pp. 522-47.

17 D.W. MeINIG [6], 205-13.

18 M. del Olmo y F. Monge [3].

R. I., $1999, \mathrm{n}^{\circ} 217$ 
niendo el «problema indio» ${ }^{19}$ y gestando una política india federal destinada a finalizar con este «problema». Las primeras formulaciones globales de una política india se inscribieron en la propia construcción del estado federal. Acostumbrados a una versión un tanto épica o cinematográfica del tratamiento de los indígenas por los mandatarios estadounidenses, se olvida ubicar la primera formulación de política indígena dentro del pensamiento ilustrado y como un producto de éste. En la definición inicial de esa política es trascendental tener en cuenta el diseño que hizo de ella un ilustrado insigne y tercer presidente (de 1800 a 1808) de los EEUU: Thomas Jefferson.

\section{La primera política india de los Estados Unidos}

La administración del presidente Jefferson pretendía alejar a la nación de los males que, según él, procedían de la excesiva densidad de población y del fuerte desequilibrio en el reparto de la fortuna. Su república ideal era más una entidad de granjeros y agricultores dispersos, unidos por una voluntad común y una serie de rutas fluviales y marítimas hacia los puertos de comercio internacional, que una nación de ciudades e industrializada. Bajo esta perspectiva resultaba evidente que los Estados Unidos necesitaban cuanto más territorio ${ }^{20}$, mejor; los nativos, por otra parte, tampoco debían ser corrompidos por los usos de los colonos, era más conveniente para ellos, mientras fueran unos seres «incompletos», mantenerlos más allá del contacto con la población blanca mayoritaria.

Los indios eran, según Jefferson, miembros, como los blancos, de una misma humanidad, eso sí, «inferiores» a los blancos en sus actuales circunstancias. El poder de la razón les decía a los herederos de la ilustración que esa inferioridad era modificable si se atacaba el entorno en el que vivían. La educación y el cristianismo les restituiría a un status semejante al del blanco. Desde ese mismo momento, el indio y su problema desaparecerían. Estas opiniones eran reforzadas, asimismo, por la constante decadencia de la población nativa, su disminución y estado de desvalimiento generado ante la radical transformación del entorno y quiebra de las formas de subsistencia tradicionales.

Durante los años siguientes, se prosiguió una política sobre esta misma base. El intercambio mutuo entre indios y blancos debía mantenerse al mínimo (había que evitar la contaminación de los malos vicios de muchos europeos). El primer encargado de la recién creada Oficina India (luego Bureau of Indian Affairs,

19 Aunque el título «problema indio» pueda resultar siniestro, en especial tras la Segunda Guerra Mundial, es necesario indicar que es una denominación surgida de la propia época.

20 En 1803 se compró por la cantidad de 15 millones de dólares la Lousiana a Francia (comprendía el territorio que se extiende desde el oeste del río Mississippi a las montañas Rocosas, exceptuando las posesiones españolas entre las que se contaba Tejas y los futuros estados de Arizona, Colorado y Nuevo México aproximadamente). 
BIA), Thomas L. McKenny, persiguió activamente una política de «emigración, preservación y mejora de los nativos ${ }^{21}$. Mientras no estuvieran preparados para comportarse como los europeos y fueran capaces de integrarse y ser asimilados en la emergente sociedad mayoritaria estadounidense, los nativos debían habitar territorios reservados para ellos, lejos de las áreas en las que los blancos se estaban asentando. Por su puesto, aunque algunos blancos fueran conscientes de ello, parecían darle poco valor al hecho que, a menudo, los territorios elegidos o «reservados» no sólo no les permitían mantener su tradicional modo de vida sino que, además, eran inadecuados para que los nativos se convirtieran en «granjeros», como de hecho iba a ocurrir con muchos de los europeos que allí se asentaron.

El progresivo desarrollo de una política indígena por parte del gobierno federal y la institucionalización de la agencia destinada a llevar a cabo dicha actividad puede considerarse, desde la perspectiva colonizadora, como el inicio formal de la política india. Se mantenía, asimismo, el instrumento del tratado como medio de incorporar legalmente a los nativos, y sobre todo sus territorios, a la soberanía del Congreso y los Estados Unidos respectivamente. Estos tratados, sin embargo, estaban sujetos tanto a crítica por parte de muchos líderes blancos como expuestos a ser rotos por el Congreso, como ocurrió en 1830 cuando se impuso el traslado forzoso ${ }^{22}$ de numerosos grupos indígenas del este del Mississippi al Territorio Indio ${ }^{23}$ designado por las autoridades de Washington, D.C., al oeste de este río. Este territorio, denominado así en 1830 bajo la presidencia de Andrew Jackson (1828-36), era una solución política ideal mientras existiera el «problema indio» ya que se evitaban así, por un lado, los conflictos territoriales con los blancos, mientras que, por el otro, se designaba una zona estable en la que llevar a cabo, bajo el control del Congreso y con la acción de la Oficina de Asuntos Indios, el proceso de asimilación que terminaría con los indios.

Dicha política contenía, en mi opinión, dos importantes paradojas: en primer lugar, se empeñaba en tratar como «indios» a una serie de grupos o naciones bien distintos, que en modo alguno se percibían a sí mismos como una sola categoría; y una segunda que era la perpetuación de un sistema de dependencia coyuntural, debido a la crisis a la que se habían visto sometidos los nativos durante la coloni-

21 McKenny había sido anteriormente el Superintendente de Comercio Indio (1816-22), institución antecesora de la Oficina India. La génesis de esta política, así como del círculo vicioso de paternalismo y dependencia, que todavía no ha llegado a romperse plenamente, se describe de un modo conciso por F.P. PRUCHA [12].

22 Este lamentable episodio, conocido como el Sendero de las Lágrimas, consistió en el traslado, a pie y en malas condiciones de las naciones nativas Cherokee, Choctaw, Chickasaw, Creek y Semínola. En dichos viajes murieron no menos de 15.000 nativos (sobre todo mujeres, niños y ancianos). Véase, Del OLMO y MoNGE [3], pp. 40-50, y WALDMAN [1], pp. 183-85

23 En 1825 el Secretario de la Guerra, John Calhoun, delineó con el apoyo del Congreso y bajo la presidencia de James Monroe un nuevo País Indio, que luego se convertiría en Territorio Indio cinco años después, WALDMAN [1], PP. 181-83. El llamado Territorio Indio continuaría transformándose durante los años hasta que, en 1907 se convirtió en Oklahoma, el 46 estado de la Unión. 
zación y que continuaba con el desarrollo de unas instituciones construidas para tratar con «indios» (atención, no distintos tipos de nativos) desde unos principios radicalmente paternalistas.

Durante las dos primeras décadas, la política de «segregación» pudo mantenerse, a pesar del progresivo crecimiento de la posición de preponderancia blanca, mediante la firma de tratados. Dicho instrumento funcionaba, pese a importantes rupturas por parte del Congreso, como es el caso de la imposición de la Ley de Relocalización India (India Removal Act, 1830) que trasladaba a los indígenas del este al oeste del Mississippi, como un acuerdo entre entidades políticas soberanas. Sin embargo, con el paso del tiempo y la apertura del «oeste» al asentamiento de colonos, estallaron una serie de guerras que establecieron un patrón de establecimiento $\mathrm{y}$, a continuación, de ruptura de los tratados mientras la expansión continuaba. El mismo Secretario Ayudante de Asuntos Indios del Departamento de Interior, Kevin Gover, afirmaba el 28 de abril de 1999 ante el Comité de Asuntos Indios del Senado que: «La Oficina de Asuntos Indios se encargó de hacer llegar a las Tribus la solemne garantía de los Estado Unidos de que sus territorios tribales serían protegidos para siempre, sólo para renegar de ese compromiso una y otra vez, así como para romper esas promesas por la fuerza de las armas» ${ }^{24}$. Los tratados no sólo se rompían sino que, además, se convertían en instrumentos de presión, cuando no de expoliación, y mecanismos diseñados, con la buena voluntad añadiría el mencionado Francis P. Prucha, para transformar a los nativos en agricultores. Sólo así, consideraban los constructores de la política india, se llegaría a la solución del problema indio gracias a su integración y disolución en los Estados Unidos. La «nación americana» discurría entonces desde un cierto deísmo ilustrado hacia el dominio de un patrón nacional fundado en los principios morales del protestantismo anglosajón.

En buena lógica, el siguiente paso con respecto a los nativos no se haría esperar mucho más, tras la Guerra Civil llegó la etapa de reconstrucción del país y, entre los programas destinados a tal efecto por el presidente Ulisses S. Grant, se diseñó una formula de trato con los nativos que buscaba activamente la pacificación. La paradoja es que este periodo es probablemente en el que se produjeron más choques armados entre blancos y nativos, y que la paz sólo llegaría con las últimas derrotas militares a finales del siglo. La oficina de comisarios indios de Grant representaba adecuadamente el nuevo espíritu que dominaba el país: desde una política ilustrada secular, Grant daba una nueva vuelta de tuerca e introducía una perspectiva filantrópica cristiana en consonancia con los principios con los que se abordó la reconstrucción. Esta oficina elaboró, sin apenas experiencia con

${ }_{24}$ El documento se encuentra a disposición del público en Internet, http://www.doi.gov/bia/ testimony/biasthm.html ( «Statement of Kevin GovER, Assistant Secretary - Indian Affairs, Department of the Interior before de Senate Committee on Indian Affairs Oversight Hearing on the Bureau of Indian Affaris and Mission April 28, 1999»). 
los nativos y nada más llegar al poder, un informe que iba a definir el plan maestro de los Estados Unidos hasta los años 1920 aproximadamente.

La solución, su solución, pasaba por la destrucción de lo que quedaba de los antiguos patrones culturales nativos. Así, con el objetivo de elevar el estatus moral del «indio» y equipararlo al del «blanco» era prioritario romper la autoridad tribal mediante una fórmula que parcelase la propiedad comunal de la tierra, que debía someterse a las leyes de los Estados Unidos y los indios convertirse en ciudadanos, gracias al desarrollo de un sistema de educación, en inglés, para los nativos.

La materialización de esta política de asimilación inducida no se haría esperar demasiado, en 1871, el Congreso decidió que no iba a firmar ningún tratado más (aunque mantenía, al menos nominalmente, su compromiso a cumplir los ya firmados). La pacificación casi total hacía ya innecesaria, según el Congreso, la política de tratados. Era evidente, también según su perspectiva, la necesidad de cambiar radicalmente el marco legal. Los objetivos a cumplir, la desaparición del «problema indio» tal como he indicado, no se estaban alcanzando. El nuevo curso aparecía ante los ojos del «Gran Padre» 25 de un modo bien visible. Los indios tenían que recibir una parcela, con título de propiedad individual, de los terrenos reservados para que pudieran cultivarla como granjeros, y no como nativos. $\mathrm{Pa}$ gos anuales que cubrieran las necesidades de educación y otros elementos «civilizatorios» serian librados por el Congreso, y se estableció un sistema de ayuda que les permitiera desarrollar las granjas (tan deseadas por los legisladores). Dichas provisiones componen el cuerpo fundamental de la nueva Ley de Parcelación General o Acta Dawes (General Allotment Act, 1887).

Los resultados del cambio de rumbo no pudieron ser más desastrosos ${ }^{26} \mathrm{y}$, de algún modo, finalizaron con las últimos reductos de vida nativa anterior al contacto. La depauperación y estado de dependencia en que se sumió a los nativos durante esos años alcanzó su cota más baja. Sólo en territorio habían perdido más de 90 millones de acres. Los hijos se «arrancaban de sus familias para enviarles a internados donde se les enseñaba el idioma, cultura, valores, hábitos, e industrias

25 El marco de relaciones entre los nativos y los blancos no sólo estaba constreñido o modificado por la superposición, como he mencionado, del concepto de indio sobre su identidad nativa sino que, además, la propia imagen que el gobierno o legisladores querían transmitir al postularse como «Gran Padre» es un mito europeo que les llevaba a comportarse, también, de un modo estereotipado. Con respecto a la construcción de mitos europeos, véase: Gananath OBEYESEKERE, The Apotheosis of Captain Cook. European Mythmaking in the Pacific, Princeton, NJ, Princeton University Press, 1992; así como la perspectiva de Marshall SAHLINS, Historical Metaphors and Mythical Realities, Ann Arbor, MI, University of Michigan Press, 1981 o su traducido Islas de Historia, Barcelona, Gedisa, 1988 (ed. orig. 1985) quién generó la apertura de una fructífera polémica entre ambos antropólogos.

26 Es interesante hacer notar, como defiende Frederick E. HoxIE en A Final Promise. The Campaign to Assimilate the Indians, 1890-1920, Cambridge, Cambridge University Press, 1989 (ed. orig. 1984), la participación de antropólogos en este proyecto.

R. I., $1999, \mathrm{n}^{\circ} 217$ 
de los americanos blancos» ${ }^{27}$. Se trataba, como se decía entonces, que los indios «dejaran la manta» y abrazaran el credo que imbuía ya la segunda revolución industrial. El círculo vicioso de paternalismo y dependencia que generaban estas actitudes y políticas se acentuó hasta tal punto que, además del daño producido sobre sus «pupilos», lo más notable fue el crecimiento de la estructura administrativa de la Oficina de Asuntos Indios. Ya no tutelaban a las naciones o los grupos tribales nativos, tenían que tutelar a cada uno de los individuos o indios.

Sin embargo, dicha política tuvo efectos inesperados. Durante estos años de máxima presión blanca, los nativos terminarían convirtiéndose en «indios» ${ }^{28}$. Gracias a los represivos internados, a su separación de las comunidades originarias unidas a la prohibición de hablar en sus lenguajes nativos o seguir sus usos tradicionales, niños de diversos grupos nativos fueron hermanándose y descubriendo que sus problemas eran los mismos. Si, como nativos, su perspectiva se reducía a su grupos de procedencia, como «indios» descubrían un patrón de tratamiento y desposesión común. Junto a esta percepción y transformación que se produjo en los sujetos del nuevo experimento civilizatorio, se dieron, además, otras circunstancias tan importantes, como la educación forzosa, que iban a facilitar la génesis de movimientos de defensa nativa, ahora ya pan-indios ${ }^{29}$. Mientras que los alumnos de los internados aprendían inglés y algunos de los rudimentos básicos con los que moverse en el mundo impuesto, otros nativos iban haciéndose conscientes no sólo de la imagen que los blancos tenían de ellos sino de los modos con los que podrían luchar si se valían de esos mismos estereotipos y de las brechas existentes en la política con la que la administración pretendía conseguir su disolución como indios, asimilándoles como nuevos ciudadanos. Curiosamente, más que hacer desaparecer a los nativos colaboraron a la revitalización de múltiples grupos nativos, así como a la asunción de una nueva y activa identidad, la de indio en la sociedad estadounidense. Se abría así un largo camino de reivindicaciones y de mejora de su posición como grupo dentro una sociedad de gran diversidad de orígenes.

La primera brecha de importancia sobre la que montar los movimientos de defensa india propiamente nativa en esta etapa surgió de un frente religioso o espiritual. Para sorpresa de los administradores, cuyo activismo cristiano era bien evidente, los indios se refugiaban en la religión, las religiones tradicionales nativas junto a los nuevos cultos de índole revitalizadora ${ }^{30}$, para la defensa de todos

27 Kevin GOVER [24].

28 Quién, en mi opinión, trata mejor esta transformación es H.W. HERTZBERG, The Search for an American Indian Identity. Modern Pan-Indian Movements, Syracuse, Syracuse University Press, 1981.

29 Anteriormente a éstos ya existían movimientos de defensa de los indígenas, como la Asociación de Derechos Indios (Indians Right Association), que se componían de blancos e indios. En estas asociaciones dominaba también y pese a todo el componente filantrópico y paternalista.

30 Los últimos estallidos de violencia o sublevación por parte de los naciones nativas de las llanuras tienen una relación directa con el desarrollo de algunos de esos movimientos revitalizado- 
sus derechos. De los múltiples movimientos que surgieron esos años quizá el más influyente fue el protagonizado por el Culto del Peyote que terminaría por dar paso, ya en 1918 a la Iglesia de los Nativos Americanos. Gracias a su impulso, así como por su influencia, surgieron numerosos movimientos pan-indios, primero, más o menos radicales y, luego, comenzó a consolidarse una conciencia común o «pan-tribu» como defiende Hertzberg [30] en su libro. La voz del indio comenzaba a sonar cada vez con más insistencia y sin ayuda de intermediario alguno. Aunque es muy difícil sintetizar la creciente actividad nativa en la sociedad estadounidense ${ }^{31}$, estaba claro que la tendencia «asimiladora» había fracasado y que una aproximación, siquiera superficial, al estado de los indios en las reservas y las ciudades confirmaba el peor de los escenarios posibles. La política inaugurada con la Ley General de Parcelación India (1887) también había fallado estrepitosamente.

\section{DEL «NUEVO TRATO» AL «AUTOGOBIERNO».}

El viejo sueño de unos Estados Unidos homogéneos y sin grandes diferencias entre sus ciudadanos estaba empezando a quebrarse también. Bajo el influjo de los propios acontecimientos históricos y, sobre todo, del gran flujo inmigratorio, parecía cada vez más claro que era imposible «homogeneizar e igualar». Las respuestas que, por otra parte, daban las ciencias sociales a este dilema no pasaban tampoco por la homogeneización, que en el caso de los indios suponía su asimilación y ulterior disolución, sino por una visión más jerárquica de la sociedad que enfatizara, como afirma $\mathrm{Hoxie}^{32}$, la coexistencia y la interacción entre los distintos grupos que poblaban el país. Los asimilacionistas perdían terreno día a día mientras que alternativas que buscaban la reforma india reivindicaban los valores intrínsecos de las culturas nativas: sus principios espirituales, su artesanía, así como una imagen más acorde con la naturaleza en un momento en que en las ciudades más destacadas del país se abarrotaban masas de inmigrantes de numerosas procedencias. Se produjo así una vuelta, por parte de algunos anglo-

res o milenaristas. Sin embargo, se insiste menos en su faceta pacífica que permitió una recuperación del orgullo por la identidad propia.

31 Además de la bibliografía a la que me he referido en la notas iniciales de este artículo, como MCNickle [3], HoXie [10], Waldman [1], Washburn [1], Del Olmo y Monge [3]. Con respecto a los movimientos religiosos, véase: F. MONGE, ««Movimientos mesiánicos e Identidad Indígena: Estados Unidos y Nueva Zelanda» Revista española de antropología americana, 15 (1985), pp. 261-81 y la tesis de grado inédita: F. MONGE, Análisis comparativo de los indígenas de Nueva Zelanda y Norteamérica, Madrid, Universidad Complutense de Madrid, 1984, en particular el capítulo 6: El nativo en el mundo blanco, pp. 151-90.

32 El libro de HoxIE [26] es un excelente análisis de la última gran campaña de asimilación de los indios (1880-1920) y de cómo los objetivos de la política india federal se transformaron en la época en que se terminó imponiendo el pensamiento «científico». 
americanos, hacia una imagen positiva, romántica y curiosa del indio. A esta época corresponden las fotos (montadas y con atuendos que ya no usaban) de artistas como E. Curtis, o los textos sobre los indígenas (tan relacionados con los defensores de los Parques Naturales en Estados Unidos y el movimiento Scout) ${ }^{33}$.

Las tres transformaciones a las que he aludido, la quiebra de los Estados Unidos homogéneos, la revitalización india y el cambio de imagen fomentado por ciertos grupos de defensa de la naturaleza y los nativos, llevaron a replantear un cambio radical de la política indígena desde, al menos, comienzos del siglo. Su mayor artífice y promotor, desde el mundo blanco, fue sin lugar a dudas John Collier, quién se convirtió a la «causa india» tras un viaje al sudoeste de los Estados Unidos en 1920. Su primera etapa como propagandista de una modificación de la actitud hacia el indio se vió acompañada por la aparición de un informe, conocido como informe Merriam (oficialmente denominado «El Problema de la Administación India»,1928), en el que se criticaban de modo exhaustivo los múltiples errores cometidos por la administración de los asuntos indios. Tomado una vez más como plan maestro, abrió una etapa de transformaciones que tuvo su culminación en el nombramiento de John Collier como Comisario de Asuntos Indios por la administración del «New Deal» del presidente Franklin Delano Roosevelt. Bajo el mandato de Collier, que se extendió hasta 1945, se aprobó en 1934 la Ley de Reoganización India (Indian Reorganization Act) que suponía un radical cambio de dirección en la política india. Se finalizaba con la política de parcelación y se entraba en un nuevo impulso legislativo que tenía como metas finales no la asimilación, sino el autogobierno y la autosuficiencia económica.

La nueva ley abría el camino a numerosas disposiciones y programas de ayuda que buscaban la incorporación del indio, desde su propia identidad nativa, a la sociedad de los Estados Unidos. Equiparación significaba ahora reubicar a los nativos, como grupo, en una posición dentro del entramado de distintas culturas y grupos étnicos que componían el país sin que ello significara la pobreza y dependencia endémica. La ley animaba a que las distintas tribus adoptaran constituciones y cartas de gobierno escritas que les permitieran autogobernarse. Establecía también un programa de créditos que les permitieran el desarrollo de programas educativos, de reorganización o de compra de terrenos propios. Animaba asimismo a la obtención de la ciudadanía por los nativos (que habían recibido técnicamente ésta a partir del año 1924).

Pese a lo prometedor de los avances, que se veían impulsados además por una mayor participación de los propios nativos, iban a surgir numerosos proble-

33 En España, esta imagen, promovida desde editoriales como Olañeta, ha irrumpido en un mercado deseoso de conocer lo «exótico» y romántico del «indio americano» y ha oscurecido la pujante vitalidad de los que sí tienen voz propia. Este fenómeno, se ha visto acompañado, por películas como «Bailando con lobos» que, haciendo uso de una buena campaña publicitaria, han querido presentar como la primera obra cinematográfica respetuosa y fidedigna sobre «indios» una visión no menos romántica, teñida de ecologismo, de los nativos de las praderas. 
mas ya que si bien es evidente que el papel de los nativos en su propio destino fue también activo (pese a la obvia desorganización y dependencia de los mismos), iban a recuperar durante este siglo un papel no soñado ni por los nativos ni sus más firmes defensores. No obstante los siguientes años iban a traer sus reveses. En primer lugar la Ley de Reorganización India (1934) tenía también importantes deficiencias, entre ellas la imposición de un sistema de gobierno basado en los principios democráticos más que los propios o tradicionales de los grupos a los que se trataba reorganizar. Por ello, pese al carácter universal de la aplicación de la misma, las nuevas medidas no fueron aceptadas por los nativos sin recelo. Bien es cierto que podían o no, por decisión propia, aceptar la aplicación de la nueva ley, pero los aspectos paternalistas y de «terminación» de la protección por parte del Congreso del patronato sobre los indios generó mucho recelo entre sus patrocinados, ya que la asunción por parte de los grupos tribales de sus propios asuntos era un modo, en su opinión, enmascarado de finalización de los compromisos asumidos por el Congreso cuando se firmaron los tratados.

Mientras tanto, el Congreso y los enemigos de la nueva política volvieron a prevalecer tras la renuncia de Collier en 1945. En 1950 se inicia un breve proceso de cambio de tendencia que establece el principio de «terminación» como el objetivo deseado. La solución, para los defensores de la terminación no pasaba por una «retribalización» de los indígenas sino por los principios de «libertad» y «emancipación» que caracterizaban, o debían caracterizar, a los ciudadanos de los Estados Unidos. No suponía, como algunos defensores de la reorganización de los nativos sugirió, un intento por integrar definitivamente y como iguales a los nativos. La política de terminación ${ }^{34}$ fue acompañada por un intenso programa de relocalización india que parecía necesario dada la falta de trabajo disponible en las reservas. El fuerte crecimiento demográfico de los nativos, por otra parte, hacía mucho más agudos y difíciles de tratar sus problemas, precisamente en el mismo momento en el que se pretendía retirar progresivamente los sistemas de ayuda y cobertura de los indios en las reservas. Así la solución perfecta parecía estimular su mudanza a las ciudades.

En los años sesenta este movimiento hacia los ciudades se había demostrado una vez más como inadecuado. Muchos nativos volvían a las reservas para acogerse a programas, contrarios a menudo al espíritu de terminación, de entrenamiento laboral. El estado de depauperación de los indios tampoco parecía atajarse demasiado bien por esta vía, así que en esta década se asiste a un nuevo cambio de tendencia por parte del gobierno federal que retorna a los principios de la ley de Reorganización.

Y llegamos, por fin, al momento clave para el relanzamiento de reivindicaciones de los indios en Estados Unidos. Con la aparición de los movimientos de lucha por los derechos civiles, los indios recobran un protagonismo largo tiempo

34 Una descripción más completa y técnica de la política india durante este siglo puede consultarse en: Lawrence C. KELLY [10].

R. I., 1999, n. $^{\circ} 217$ 
perdido. En un clima social turbulento, son capaces de realizar una serie de golpes de efecto importantes, como son la toma de la Isla de Alcatraz en 1969, la Marcha de los Tratados Rotos a Washington, organizada por al Movimiento del Indio Americano (American Indian Movement), que finalizó con la toma y destrucción de la Oficina de Asuntos Indios, y la también toma de Wounded Knee. Estas protestas terminaron por inclinar la balanza en favor de algunas de sus reivindicaciones y provocaron una actitud más receptiva por parte de la administración. Sensibilidad que, por otra parte, estaba en sintonía con el espíritu que pretendía insuflar la Ley de Reorganización India.

La historia india ofrece numerosas paradojas para aquellos que se acercan a ella conociendo la de los Estados Unidos. Así, la presidencia del controvertido Richard Nixon es la que genera el impulso transformador que todavía hoy impera en la política india de los Estados Unidos. Nixon formuló en un mensaje sobre política india al Congreso el lema que presidiría el último cuarto de este siglo: autodeterminación sin terminación. Se combinaban así dos principios hasta entonces considerados como opuestos, ya que se pretendía ahora aunar una política de patronato indefinida con otra que trasladara a los indígenas una progresiva capacidad de autogestión. Estos principios se transformaron en una ley de Autodeterminación y Asistencia a la Educación (Indian Self-Determination and Education Assistance Act, 1975) que pretendía resolver las tensiones y conflictos de ambos espíritus.

El resultado de la ley ha sido hasta ahora variado, ya que mantiene una contradicción en sí misma ¿Cómo es posible autogobernarse cuando se mantiene un sistema de protectorado sobre los indios? Los fondos y los propios proyectos de los nativos están sujetos a la fiscalización y normativa federal. Las colisiones son constantes y han generado un fenómeno nuevo en la formulación de la política indígena. Los nativos ya no son los derrotados restos de las culturas supervivientes a la conquista, tras su renovada revitalización, sino que mantienen un complejo sistema cultural de nuevos y viejos principios.

Ellos mismos se enfrentan a importantes dilemas relacionados con su propia acción, por ejemplo sobre la educación que pueden ahora promover. Algunos, como la kwa-kwa-ka' wakw Nella Nelson, piensan que tanto los nativos como los no-nativos deben aprender lo mejor de las dos culturas. Otros, como un profesor del grupo Makah del estado Washington, señalan las contradicciones y dilemas en los que se mueve su propia acción:

«Para hacer que un indio cumpla con los principios europeos de comportamiento social se hizo necesario separarle de sus propios valores, que se habían propagado exitosamente durante miles de años. La máxima amenaza [existente] era individualizar el pensamiento de cada persona india, separarle de sus tendencias naturales hacia el pluralismo.

El sistema de escuelas nació de la Revolución Industrial. Su filosofía parece ser la de ofrecer un producto que se adecue a las máquinas industriales de 
nuestros tiempos. Con lo que se fortalece la idea de tratar de seguir carreras [características de la sociedad mayoritaria].

Pero hay muy pocas carreras posibles en las reservas indias. Los intentos de continuar el sistema educativo con estos principios incrementan la posibilidad de conflicto. Si un niño indio va a abandonar sus valores tal como le fueron transmitidos a él de generación en generación ..., debe entonces estar preparado para aceptar las consecuencias de su decisión. Esto es algo extremadamente difícil de hacer, especialmente por culpa de los aspectos legales por los que está limitado. Él es todavía un indio, con una relación definida con su tribu.

La misma promesa de la democracia es contraria al tribalismo ya que aboga por un respeto y preocupación de los individuos desde su nacimiento. Las perspectivas de los tribalistas son más de auto-sacrificio, de una auto-realización con estrechas relaciones con la familia y la comunidad. Si la promesa de una educación óptima significa que enseñar y aprender debe individualizarse ..., las escuelas entonces deben reconsiderar su posición. Siendo indios tenemos valores distintos de la sociedad no-india, una diferente filosofía de vida, distintas aspiraciones en la vida y la posibilidad real de tener distintos objetivos ${ }^{35}$.

Y luchan frente a la administración y, a veces, la sociedad mayoritaria, para defender o imponer sus propias interpretaciones sobre los límites que sus derechos inalienables, los tratados y la normativa legal vigente les impone. Emprenden, para ello, acciones legales que van clarificando poco a poco su margen de maniobra. Gracias a estos litigios hoy se reconoce que los poderes de que gozan en los Estados Unidos no proceden de la delegación de los mismos hecha por el Congreso, sino de su propia, aunque limitada, soberanía. Los indios hoy son ciudadanos de los Estados Unidos que conservan una serie de derechos que son anteriores a los propios Estados Unidos. Mientras que el Congreso no se pronuncie ellos mantienen numerosos privilegios y posibilidades ocultas de obtener ventajas y derechos en su propio favor, como ha sido el caso durante los últimos años.

Un hito importante en la afirmación de los derechos de los nativos ha sido, sin lugar a dudas, el acuerdo conseguido entre los Estados Unidos y los nativos de Alaska en 1971. Desde que los Estados Unidos compraron Alaska a Rusia en 1867 se ha mantenido un contencioso sobre las reclamaciones de tierras nativas. Al fin, con la Ley de Acuerdo sobre las Reclamaciones de Nativos de Alaska (Alaska Native Claims Settlement Act, 1971), se les reconocían cuarenta millones de acres de terreno de su propiedad, se les compensaba con una cantidad cercana a los mil millones de dólares y se instituían corporaciones nativas destinadas a mantener sus activos o bienes.

Las luchas y conflictos continúan y continuarán sin duda. Nuevas «brechas» legales no percibidas por el Congreso o la reinterpretación de sus derechos reconoci-

35 Ruth KIRK, Tradition and Change on the Northwest Coast. The Makah, Nuu-chah-nulth, Southern Kwakiutl and Nuxalk, Seattle, WA, University of Washington Press, 1986, pp. 246-47.

R. I., 1999, n. ${ }^{\circ} 217$ 
dos, les ha permitido, por ejemplo, levantar multimillonarios complejos turísticos asociados a casinos de su propiedad, fundar prósperos negocios de venta de productos, como tabaco, libres de impuestos en algunos estados (con la consiguiente indignación por parte de ciudadanos y políticos desconocedores de los derechos históricos que asisten a los nativos a la hora de mostrar su propio espíritu emprendedor).

Aunque para el Congreso todavía es hoy técnicamente posible desmontar lo mucho conseguido por los indios, parece que a estas alturas la «marcha atrás» es imposible. Los nativos han sabido conquistar numerosos «territorios» vacantes y comenzar a labrarse una imagen y posición menos marginal en el nuevo modelo de sociedad multicultural que pretende impulsarse en los Estados Unidos. No es de extrañar que en este clima florezcan «nuevos» artistas en música o literatura que mezclan lo nuevo y lo viejo del mundo nativo, en unos productos culturales que no sólo circulan entre ellos, sino que cuentan con una penetración cada vez mayor en el mercado tanto nacional como internacional. Hoy no es difícil encontrar una sección de música india actual en las estanterías de las tiendas de discos, ni leer traducidos autores que abordan temáticas o preceden del mundo indio, como es el caso de la novelista Louise Erdrich ${ }^{36}$.

Sin embargo, lo que considero como un síntoma más esperanzador de la nueva posición del «indio» en la sociedad y cultura estadounidense no es su presencia a través de las actividades económicas sino, sobre todo, su progresiva aparición como «caricatura» o «ciudadano» en las comedias de situación y series televisivas (como, por ejemplo, «Doctor en Alaska» -'Northern Exposure-). Parece que, con la desaparición de la «transparencia» de la que gozaba, su propio estereotipo es objeto de broma a nivel popular y que, con ello, sean los propios nativos los que nos expliquen ellos mismo sus perspectivas.

El «siglo corto» que ahora estamos abandonando ha sido, desde que se produjo el llamado descubrimiento de América, el que ha visto una recuperación de los nativos más espectacular. Las sombras y los problemas, la marginación y el desempleo son todavía hoy graves problemas para los nativos de los Estados Unidos, sin embargo, por primera vez desde que los europeos pusieron su pie en ese continente se vislumbra la esperanza y la renacida capacidad de los nativos americanos por ser dueños de su propio destino.

Devised as an introductory survey of the Unites States Indian Policy, this paper is also an essay which intends to show such Indian policy as a by-product of the historical context as well as of the origin, evolution and construction of the Country.

36 Louise ERDRICH ha publicado las novelas traducidas al español, La Reina de la remolacha (The Beet Queen), Medicina de Amor (Love Medicine), Pistas (Tracks) y El Palacio de Bingo (The Bingo Palace) sobre los problemas y experiencias de los nativos americanos en el medio oeste. 